\title{
GaN-based Semiconductor devices with Multichannel Structures
}

\author{
Snehal Koparde \\ Asst. Professor, Marathwada Mitra Mandal's College of Engineering Pune India \\ snehalkoparde@mmcoe.edu.in
}

\begin{tabular}{|l|l|}
\hline Article History & \multicolumn{1}{|c|}{ Abstract } \\
$\begin{array}{l}\text { Article Submission } \\
\text { 04 October 2021 } \\
\text { Revised Submission } \\
11 \text { November 2021 } \\
\text { Article Accepted } \\
10 \text { December 2021 } \\
\text { Article Published }\end{array} \quad \begin{array}{l}\text { Semiconductor junctions, for example, pn-intersection and Schottky intersection have } \\
\text { been very significant as essential components for different semiconductor gadgets. } \\
\text { The break-down voltage of the junction PN-intersection relies upon the polluting } \\
\text { influence fixations in semiconductors. An opposite voltage is supported in the } \\
\text { exhaustion layer thickness which is corresponding to the square of base voltage. The } \\
\text { breakdown happens, when the electric field with an intersection arrives at the } \\
\text { semiconductor regardless of whether the consumption layer can be extended } \\
\text { substantially. To accomplish higher go down voltage, a wide band hole } \\
\text { semiconductor, for example, silicon carbide has been examined. The electron gadgets } \\
\text { utilizing silicon carbide may undergo serious issues. In any case, the breakdown } \\
\text { system of the intersection utilizing silicon carbide is as yet equivalent to that of the } \\
\text { conventional junctions. As of late, GaN utilized electron gadgets have developed as } \\
\text { cutting-edge high force exchanging gadgets inferable from their high breakdown } \\
\text { field. A few papers have revealed that GaN-based HFETs is directly expanded by } \\
\text { expanding the junction dividing between the door and channel electrodes. This } \\
\text { conduct is unique in relation to that of the customary junctions. } \\
\text { Keywords: Semiconductor junctions, Bandgap, GaN material, electrodes. }\end{array}$ \\
\hline
\end{tabular}

\section{Introduction}

Sensational increment of portable correspondence frameworks has extended the field of intensity semiconductor gadgets. Those gadgets must be worked at microwave recurrence from a few hundred $\mathrm{MHz}$ to a few $\mathrm{GHz}$ ranges, where compound semiconductor gadgets are generally fit. A compound semiconductor material spoke to by GaAs has better effectiveness than silicon since compound semiconductor materials accomplish low RF misfortune that originates from the entirety of DC misfortune (obstruction misfortune) and AC misfortune (capacitance misfortune). The first starts from their high electron portability and the other from semi-protecting qualities of the wide band hole materials. This inalienable nature of wide band hole likewise gives them higher breakdown voltage attributes than silicon, which is additionally significant when the gadgets are downsized. As for the mobile phones, productivity is a significant concern since they must be worked by the battery-powered battery whose capacity limit is constrained. RF power gadgets are divided into two significant application classes. One is the versatile terminals (Power go $1-4 \mathrm{~W}$ ) where the high-proficiency and low voltage activity are required, the other is base stations (Power run 20-400W) where high yield power with adequate linearity is required.

Table 1: Characteristics parameters of Si, SiC and GaN Semiconductor Materials

\begin{tabular}{|c|c|c|c|}
\hline Parameters & Si & SiC & GaN \\
\hline Bandgap (eV) & 1.1 & 3.3 & 4.4 \\
\hline Electron Mobility(cm $\mathbf{2} / \mathbf{V s})$ & 1300 & 780 & 1500 \\
\hline Electric field for breakdown (MV/cm) & 0.3 & 3 & 3.3 \\
\hline Figure of Merit & 1 & 4 & 11 \\
\hline
\end{tabular}

It is noticed that the modern adjustment frameworks spoke to by QPSK or OFDM require very direct force activity so as to oblige however many supporters as could be allowed in the constrained data transmission. The 
feasible option for the last one is the nitride semiconductor materials that would achieve right around multiple times higher current voltage item. The motivation behind this paper is to survey the compound semiconductor power gadgets for correspondence framework applications. In view of the model, the on-opposition of the diodes has been definitely diminished by expanding the AlGaN thickness or the channel number of diodes, holding amazingly high separate voltages more than $9000 \mathrm{~V}$.

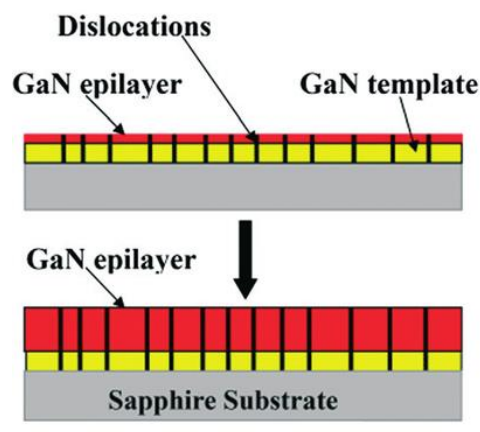

(a)

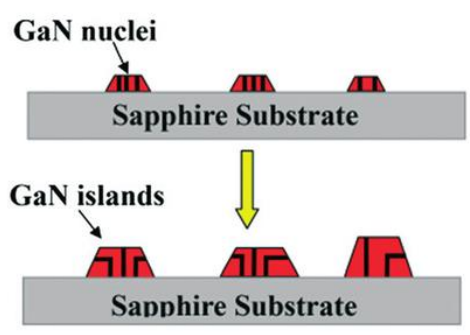

(c)

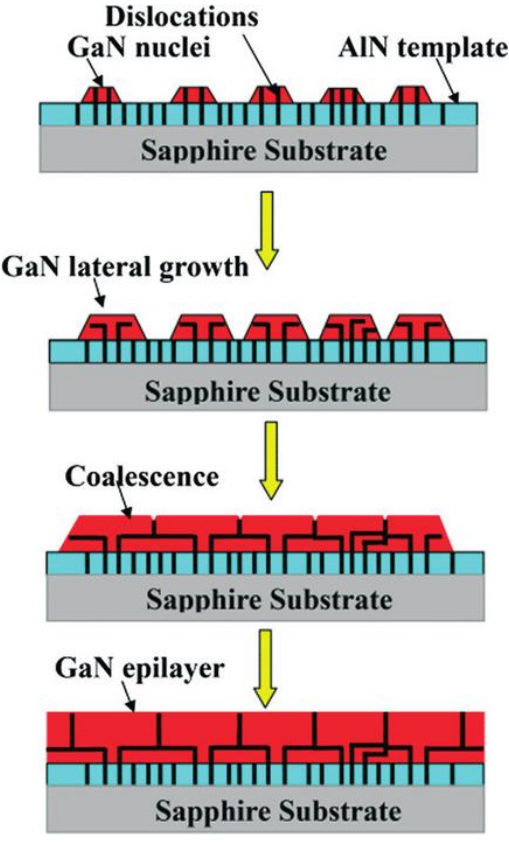

(b)

Figure 1: GaN material-based semiconductor fabrication

The high electron versatility got by two-dimensional electron gas structures with expanded sheet transporter fixation. So as to diminish the on-obstruction, shortening the channel length is best, in any case, the methodology winds up in expanding the entryway opposition that falls apart the RF gain. Other than this, shortening channel causes higher channel conductance that likewise corrupts the force gain and accordingly the helpless Power-Added Efficiency (PAE). High breakdown voltage is required for the base stations on the grounds that the lower power misfortune is reachable by utilizing bigger voltage swing as opposed to bigger current swing in high influence transmitters. To expand the breakdown voltage of the GaAs FETs, decreases of the doping fixation or potentially diminishing the channel thickness are significant. It is notable that the GaAs or $\mathrm{AlGaAs}$ surface influences the channel current of FETs as the activity recurrence increments. This recurrence scattering is because of the transporter catching procedure where electron discharge is slower than catching, which winds up in charging surface negative potential. This impact causes the genuine bending issues of GaAs FETs. The current twofold break structure is viable to smother such deplete current debasement by finding the channel well beneath the semiconductor surface. Breakdown voltage of the present MODFET is unequivocally subject to counter balance length just as the break depth.

\section{References}

[1] V. E. Ilian et al., "Evaluation of semiconductor devices aging from in core flux detector amplifier used in nuclear power plants equipment," Proceedings. International Semiconductor Conference, Sinaia, Romania, 2002, pp. 355-358 vol.2, doi: 10.1109/SMICND.2002.1105867.

[2] P. Szabo, A. Poppe and M. Rencz, "Studies on the Possibilities of In-Line Die Attach Characterization of Semiconductor Devices," 2007 9th Electronics Packaging Technology Conference, Singapore, 2007, pp. 779-784, doi: 10.1109/EPTC.2007.4469707.

[3] Z. Guo, X. Tu, J. Pan and F. Lu, "ESD Models and Measurement for Semiconductor Device," 2006 7th International Conference on Electronic Packaging Technology, Shanghai, 2006, pp. 1-7, doi: 10.1109/ICEPT.2006.359859. 